\title{
MODELAGEM E SIMULAÇÃO DE CONDIÇÕES DE OPERAÇÃO DE UMA COLUNA CROMATOGRÁFICA PARA SEPARAÇÃO DOS ENANTIÔMEROS DO PRAZIQUANTEL.
}

G. C. R. PEDREIRA ${ }^{1}$, A. G. BARRETO JÚNIOR ${ }^{2}$, A. R. SECCHI ${ }^{3}$ e M. B. de SOUZA $\mathrm{JUNIOR}^{2}$

${ }^{1}$ Universidade Federal do Rio de Janeiro, Escola de Química

${ }^{2}$ Universidade Federal do Rio de Janeiro, Escola de Química, Departamento de Engenharia

Química

${ }^{3}$ Universidade Federal do Rio de Janeiro, Escola de Química, Programa de Engenharia

Química

E-mail para contato: gi.ronze@gmail.com

\begin{abstract}
RESUMO - Os esforços focados na área de modelagem e simulação cromatográfica são essenciais para o avanço dos estudos sobre separação dos enantiômeros do Praziquantel (PZQ: medicamento que atua no tratamento da esquistossomose) de forma mais eficiente e econômica. $\mathrm{O}$ presente trabalho tem como abordagem inicial a modelagem de uma coluna cromatográfica, viabilizando o entendimento e a obtenção de conhecimento geral sobre o processo e a posterior análise do comportamento de suas variáveis. O modelo computacional implementado em Fortran será avaliado por resultados experimentais obtidos através das perturbações nas condições de operação. Apesar do processo industrial ser realizado sob a forma de várias colunas integradas em um Leito Móvel Simulado (SMB), os resultados obtidos para uma única coluna são capazes de fornecer a base para a estimação de parâmetros da isoterma de equilíbrio, valores fundamentais para a descrição do processo de separação. O presente trabalho fornece uma boa estimativa para o número de Péclet, sendo este, 1300. Além disso, analisa o efeito de diferentes concentrações iniciais e discute os dados obtidos por simulação considerando aspectos relevantes, como a sobreposição de bandas e limite de detecção dos equipamentos. Com isso, o trabalho apresenta valores de condições de operação satisfatórias para a separação dos enantiômeros do PZQ em uma coluna cromatográfica de $1,0 \mathrm{~cm}$ de diâmetro e $25,0 \mathrm{~cm}$ de comprimento, cuja fase estacionária é a celulose.
\end{abstract}

\section{INTRODUÇÃO}

\subsection{O Praziquantel}

O Praziquantel (PQZ) é um fármaco utilizado para o tratamento da esquistossomose, doença negligenciada que atinge cerca de 200 milhões de pessoas mundialmente. Apesar do PZQ tratar a doença, sua ingestão é muito difícil especialmente em crianças devido ao seu gosto amargo que provoca vômito. 
Quimicamente, sabe-se que o PZQ é um composto quiral e que seu enantiômero R atua no combate à doença (Liu, 2005). Além disso, estudos de Meyer et al. (2009) atribuem grande parcela do sabor amargo ao enantiômero S. Com isso, a motivação do presente trabalho é a separação dos enantiômeros, o que reduziria as doses de PZQ tomadas e facilitaria a ingestão e biodisponibilidade do medicamento no organismo. O estudo de um processo de separação industrialmente viável também impulsiona o presente trabalho, uma vez que apenas dessa forma o medicamento poderá ser amplamente distribuído e acessado por todos aqueles que precisam.

\subsection{Processo Cromatográfico}

A cromatografia líquida de alta performance (HPLC) permite a separação dos enantiômeros, pois devido a sua quiralidade eles interagem de forma diferente com a fase estacionária quiral, apresentando diferentes tempos de retenção na coluna cromatográfica. A mistura racêmica solvatada é injetada na entrada da coluna. Na saída, os enantiômeros saem através de duas frentes de massa diferentes. $\mathrm{Na}$ coluna estudada nesse trabalho, a primeira frente de massa corresponde ao enantiômero S. Posteriormente, ocorre a saída do enantiômero desejado, o R-PZQ.

\section{OBJETIVOS}

O presente trabalho objetiva analisar por modelagem e simulação o comportamento da separação dos enantiômeros do PZQ em uma coluna cromatográfica cuja fase estacionária é celulose, de 1,0 cm de diâmetro e $25,0 \mathrm{~cm}$ de comprimento, visando a otimização. Tal análise é feita avaliando diferentes condições de operação e utilizando parâmetros dos estudos experimentais de Farias, 2013.

\section{MODELAGEM DE UMA COLUNA}

\subsection{Modelo Fenomenológico}

Para a modelagem de uma coluna cromatográfica, descreve-se o balanço de massa num volume diferencial para cada componente $i(i=1$ representa o S-PZQ e $i=2$ representa o R-PZQ) levando em consideração as concentrações dos componentes nas fases líquida e sólida, a porosidade da coluna e o fluxo de massa. Consideraram-se relevantes para os termos de fluxo as parcelas advectiva e difusiva. De forma genérica, tem-se que:

$$
\left\{\begin{array}{c}
\text { Entrada } \\
d e i \\
\text { em } d V
\end{array}\right\}-\left\{\begin{array}{c}
\text { Sa } \tilde{\mathrm{A}}-\mathrm{da} \\
\text { de } i \\
\text { em } d V
\end{array}\right\} \hat{\mathrm{A}} \pm\left\{\begin{array}{c}
\text { Gerado } \\
\text { ou } \\
\text { consumido } \\
\text { de } \text { i em } d V
\end{array}\right\}=\left\{\begin{array}{c}
\text { Fluxo } \\
\text { advectivo }
\end{array}\right\}+\left\{\begin{array}{c}
\text { Fluxo } \\
\text { difusivo }
\end{array}\right\}
$$

Apresenta-se a seguir a forma desenvolvida da equação 1: 


$$
\frac{\hat{\mathrm{a}}^{\wedge}, c_{i}}{\hat{\mathrm{a}}^{\wedge}, t}+\left(\frac{1-\hat{\mathrm{I}} \mu}{\hat{\mathrm{I}} \mu} \hat{\mathrm{A}}\right) \frac{\hat{\mathrm{a}}^{\wedge}, q_{i}}{\hat{\mathrm{a}}^{\wedge}, t}+u \frac{\hat{\mathrm{a}}^{\wedge}, c_{i}}{\hat{\mathrm{a}}^{\wedge}, z}-D_{a x} \frac{\hat{\mathrm{a}}^{\hat{}^{2},} c_{i}}{\hat{\mathrm{a}}^{\wedge}, z^{2}}=0
$$

Onde ci e qi são as concentrações do componente i na fase líquida e na fase sólida respectivamente, $\varepsilon$ é a porosidade da coluna, u é a velocidade intersticial da fase móvel, Dax é o coeficiente de difusão, t é a variável temporal e z é a variável espacial.

No intuito de montar um sistema e encontrar a variável ci da equação 2 , é preciso utilizar uma equação da forma $\mathrm{q}=\mathrm{f}(\mathrm{c})$. Esta é a equação da isoterma de equilíbrio. Para efeitos de modelagem de uma coluna, foram utilizados parâmetros previamente estimados por Farias (2013) conforme se observa a seguir:

$$
q_{i}=\frac{a_{i} c_{i}}{1+b_{1} c_{1}+b_{2} c_{2}}
$$

Onde $\mathrm{a} 1=4,8924 ; \mathrm{a} 2=6,7466 ; \mathrm{b} 1=1,0000 \mathrm{E}-14 ; \mathrm{b} 2=0,039$.

Além disso, condições iniciais (concentrações iguais a zero em qualquer ponto da coluna) e de contorno (continuidade e definição de um degrau na entrada e saída da coluna) foram necessárias para finalizar a proposição do sistema, formado pelas equações 2 (Equação Diferencial Parcial) e 3 (equação algébrica).

\subsection{Solução numérica e modelo computacional}

Sendo muito complexa a solução analítica desse sistema, a forma de solução escolhida foi aproximação parabólica em elementos finitos, desenvolvida por Biscaia (2005). Esse método apresenta solução numérica com ótimo desempenho para a tarefa que lhe é designada neste trabalho. Através da escolha do número de elementos da discretização no método, obtêm-se dados mais precisos. Para resolver o problema de integração temporal foi utilizado um código já amplamente difundido chamado DASSL: (Petzold, 1989), desenvolvido em Fortran. Uma das atividades desenvolvidas nesse trabalho foi aprender a utilizar essa ferramenta através de uma workspace criada no Fortran com a descrição do modelo cromatográfico em diversos arquivos relacionados.

Uma vez que o modelo começou a responder satisfatoriamente recebendo condições de operação e devolvendo concentrações de cada componente em cada tempo, tornou-se possível fazer testes com a modificação de diversas variáveis.

\section{Resultados e discussão}


A análise do número adimensional Peclet, definido pela Equação 4, ilustra a predominância do efeito de advecção em relação ao de difusão no processo cromatográfico e vice-versa.

$$
P e=\frac{\operatorname{advec\tilde {A}\S \tilde {A}£o}}{\text { difusÃ€o }}=\frac{u_{i n t} L_{c}}{D_{a x}}=2 * N r
$$

Onde Lc é o comprimento da coluna, uint é a velocidade intersticial da fase móvel e Dax é o coeficiente de difusão e Nr são os números de pratos teóricos. Pela Figura 1, observase que o favorecimento da difusão provoca alargamento das frentes de massa e diminuição do pico do cromatograma. Situações de alta difusão costumam ser evitadas em indústrias onde se operam cromatógrafos, pois diminuem a concentração do enantiômero obtida ao final da coluna e além disso, aproximam seus tempos de retenção, o que pode atrapalhar sua separação. Por sua vez, quando a advecção é favorecida, é possível notar um afastamento cada vez maior entre as bandas. A predominância do efeito de advecção é acompanhada do aumento de número de pratos teóricos. Dessa forma, a Figura 1(b) representa o melhor Peclet dentre os quatro mostrados, pois neles não se observam sobreposições de banda nem saturações. Apresentando um número adimensional $\mathrm{Pe}=1300$, o número de pratos teóricos, além disso, não é tão alto.

Figura 1 - Concentrações adimensionais $(\mathrm{C} / \mathrm{C} 0)$ de cada enantiômero versus tempo adimensional ( $\mathrm{t} / \mathrm{t}$ int $)$ na saída da coluna cromatográfica variando o número adimensional Peclet. (a) $\mathrm{Pe}=$ 4000. (b) $\mathrm{Pe}=1300$. (c) $\mathrm{Pe}=440$. (d) $\mathrm{Pe}=120$ 


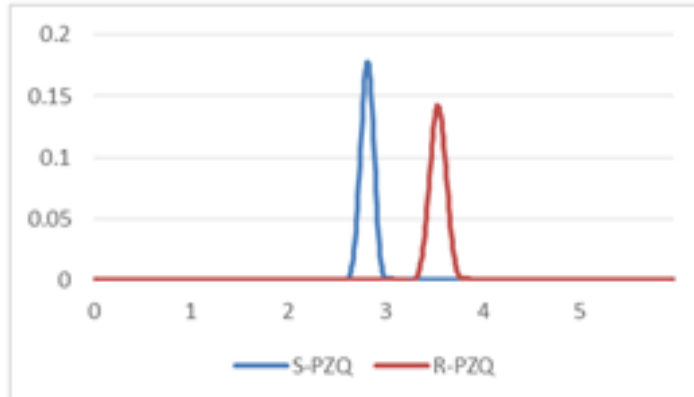

(a)

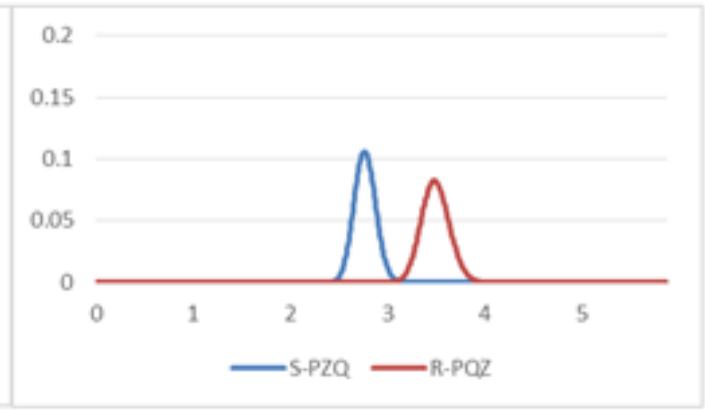

(b)

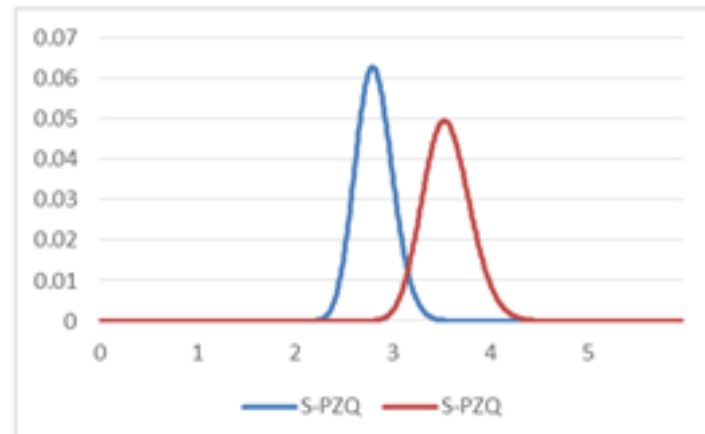

(c)

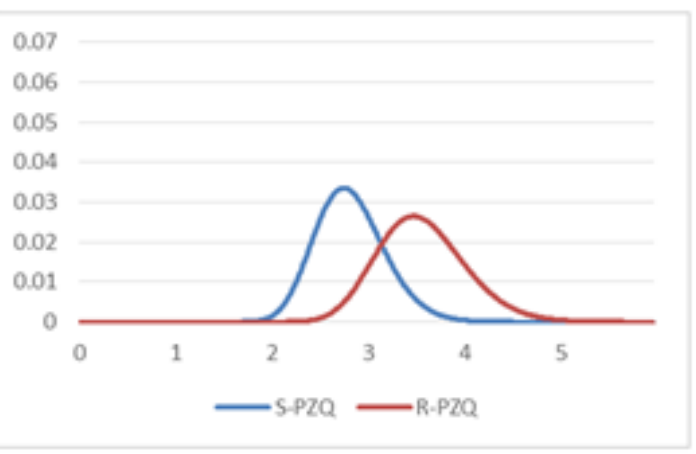

(d)

O efeito da concentração inicial no cromatograma no final da coluna considerando um Péclet igual a 1300 pode ser observado pela Figura 2 a seguir:

Figura 2 - Concentrações (em g/L) de cada enantiômero versus tempo (min) na saída da coluna cromatográfica. (a) Concentração inicial de $20 \mathrm{~g} / \mathrm{L}$. (b) Concentração inicial de $40 \mathrm{~g} / \mathrm{L}$. (c) Concentração inicial de $80 \mathrm{~g} / \mathrm{L}$.

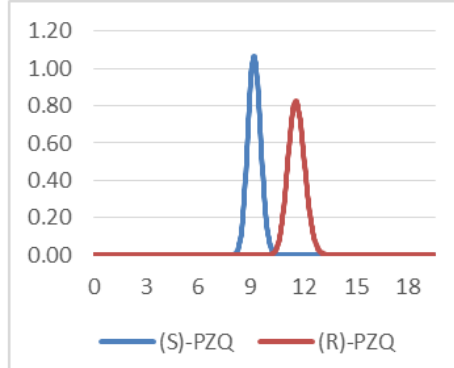

(a)

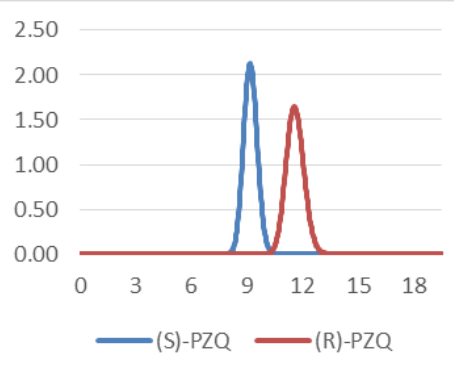

(b)

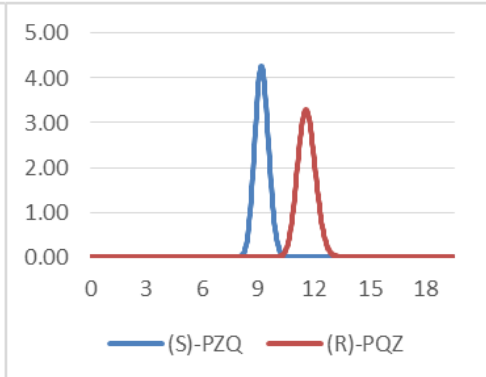

(c)

A concentração inicial de $20 \mathrm{~g} / \mathrm{L}(10 \mathrm{~g} / \mathrm{L}$ de cada enantiômero $)$ apresenta um cromatograma desejado para separação. Se a concentração inicial é dobrada ou até mesmo quadriplicada, não há alterações perceptíveis na forma dos gráficos obtidos, exceto a multiplicação do eixo y (concentrações) por um fator de escala relacionado à concentração inicial. O significado desse fenômeno é que se injetarmos uma concentração de $40 \mathrm{~g} / \mathrm{L}$ iniciais, obteremos no final da coluna amostras com o dobro da concentração de cada 


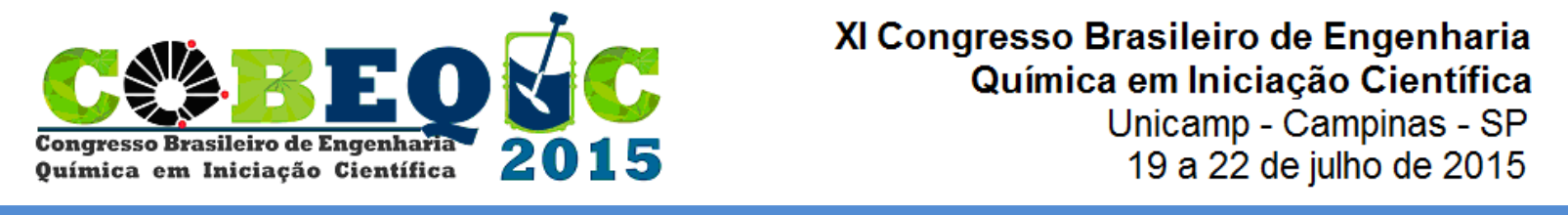

enantiômero em relação ao que seria obtido com uma concentração inicial de $20 \mathrm{~g} / \mathrm{L}$. Dos gráficos 2(a), 2(b) e 2(c), pode-se concluir que a concentração inicial no cromatograma não acarreta em efeitos de sobreposição de bandas nas condições de operação (Peclet $=1300$, vazão $=1 \mathrm{ml} / \mathrm{min}$, volume injetado $=100 \mu \mathrm{L}$ ) mesmo em concentrações iniciais consideradas altas para o Praziquantel, como $80 \mathrm{~g} / \mathrm{L}$. Como resultado, nessas condições, diferentes concentrações iniciais não irão afetar a capacidade de separação dos enantiômeros. Vale a pena ressaltar que concentrações muito baixas ou muito altas devem ser pensadas com mais cuidado antes de serem executadas na prática devido aos limites de detecção dos equipamentos, mesmo com resultados do modelo computacional satisfatórios.

\section{CONCLUSÃO}

Através das simulações realizadas no modelo Fortran, chegou-se ao resultado de que para a coluna em questão, considerando sua geometria e empacotamento da fase sólida, operar com o número de Péclet 1300 é uma condição satisfatória para separação dos enantiômeros do Praziquantel. Além disso, concentrações de $10 \mathrm{~g} / 1$ de cada enantiômero também são condições satisfatórias nessa operação em que a vazão da fase fluida é $1 \mathrm{~cm}^{3} / \mathrm{min}$ e o volume injetado na coluna cromatográfica é de $0,1 \mathrm{ml}$.

\section{REFERÊNCIAS}

LIU Y-H, Levo-Praziquantel versus Praziquantel in experimental and clinical treatment of schistosomiases Japonica. Chin Med J 106(8): 593-596., 2005.

MEYERS, T.; SEKLJIC, H.,; FUCHS, S.; BOTHE, H.; TASTE, A. New Incentive to Switch to (R)-Praziquantel in Schistosomiasis Treatment, Negleted Tropical Diseases, 3, 1, 2009.

FARIAS, B. V., Avaliação do equilíbrio de adsorção e projeto de condições de separação de praziquantel por cromatografia líquida de alta eficiência (CLAE), Dissertação de mestrado, UFC, 2013.

BISCAIA, E., Notas de aulas da disciplina Métodos Numéricos para Sistemas Algébricos e Diferenciais, PEQ/COPPE/UFRJ, 2005.

PETZOLD, L. R., 1989, DASSL code, version 1989, Computing and Mathematics Research Division, Lawrence Livermore National Laboratory, L316, PO Box 808, Livermore, CA 94559. 